\title{
Transbronchial Lung Cryobiopsy: New Options for a New Reality
}

\author{
Alfonso Torrego $^{\text {a }}$ Pallav L. Shah ${ }^{\mathrm{b}}$ \\ a Bronchoscopy Unit, Respiratory Department, University Hospital de la Santa Creu i Sant Pau, Barcelona, Spain; \\ ${ }^{b}$ National Institute for Health Research Unit, Royal Brompton \& Harefield NHS Foundation Trust, Imperial College, \\ London, UK
}

Transbronchial lung cryobiopsy (TBLC) is a new bronchoscopic procedure, which is able to improve the specimen size and diagnostic yield in comparison with transbronchial lung biopsy (TBLB) performed with a conventional forceps [1-7]. TBLB aims to obtain samples from lung parenchyma and is indicated in patients with pulmonary infiltrates or peripheral consolidations, selected according to clinical and radiological information.

Cryoprobes are used with a flexible bronchoscope and work by the Joulen-Thomson effect that occurs when a quick gas decompression generates a very low temperature compared to that of the atmosphere. The cryosystem (Erbokryo ${ }^{\circledR}$; ERBE Elektromedizin $\mathrm{GmbH}$, Tübingen, Germany) works with carbon dioxide or nitrous oxide. The fast cooling effect at the tip of the probe firmly attaches to the surrounding tissue in few seconds. Both probe and bronchoscope are then pulled out of the airways. Flexible probes 1.9 and $2.4 \mathrm{~mm}$ in diameter are available for cryotherapy and cryobiopsies.

Different studies have demonstrated that TBLC is a feasible technique in the hands of trained bronchoscopists and may become a viable alternative to surgical lung biopsy [1-7]. In addition, benefits observed from the use of cryoprobes include better sample preservation with less artefacts and crushing effects. This in turns improves the number of the specific histological patterns identified. Cryobiopsies also demonstrate that the fast freezing and thawing process preserves the capacity for molecular and staining studies $[4,8,9]$.

\section{KARGER}

(c) 2016 S. Karger AG, Basel

0025-7931/16/0913-0204\$39.50/0

E-Mail karger@karger.com

www.karger.com/res
However, a multidisciplinary approach and the diagnostic consensus reached by pathologists, clinicians and radiologists are still crucial in the management of this complex group of lung diseases [10]. In addition, TBLC still has several challenges to overcome.

Firstly, published studies show a variable incidence of complications, mostly bleeding and pneumothoraces. In some articles, the safety profile is comparable to that of the conventional biopsy forceps, but a higher rate of pneumothoraces and severe haemorrhage has also been described with this technique [3, 4, 11]. Pajares et al. [4], in a randomized trial comparing cryoprobes and forceps in patients with interstitial lung diseases inconsistent with usual interstitial pneumonia, found a higher diagnostic yield in the cryobiopsy group, even after histological findings had been submitted to a multidisciplinary approach. The same study found a very low rate of complications, with no differences in pneumothorax and no clinically relevant differences in the percentage of patients experiencing a pulmonary haemorrhage. Casoni et al. [3] described a high rate of pneumothorax (28\%) probably associated with the cryoprobe position (closer distance to the pleura) in subjects with a radiological usual interstitial pneumonia pattern (histologically confirmed in $75 \%$ of the patients who underwent a cryobiopsy).

Secondly, TBLC requires standardization. Recently, an interesting editorial by Poletti and Hetzel [12] has highlighted that the variety of modalities, i.e., cooling agents, freezing time and distance from the pleura, is

Dr. Pallav L. Shah

Royal Brompton Hospital

Sydney Street

London, SW3 6NP (UK)

E-Mail pallav.shah@imperial.ac.uk 
slightly different in the published studies. In addition, bronchoscopy settings [type of ventilation, type of airway access (tracheal tube vs. laryngeal mask; flexible vs. rigid scope) and probe diameter], radiological selection and sampling protocol (number and location of biopsies) also vary in different studies. Finally, most of these studies are observational, have been carried out in single centres and include a limited number of patients.

As mentioned before, one of the most important concerns regarding TBLC is the risk of bleeding. Cryobiopsies are too big to pass through the working channel. The bronchoscope is removed together with the cryoprobe once the biopsy is obtained. Hence, there is loss of visual contact with no option to place the bronchoscope in the segment immediately after the biopsy to collapse and suck a possible haemorrhage. In the first TBLC studies, a second bronchoscope was ready and inserted immediately after biopsy had been used to keep up visual airway control, but this may still be inadequate in the context of a massive bleeding $[1,2]$.

Some recent studies use a bronchial haemostatic blocker (Fogarty balloon or equivalent) placed routinely before any cryobiopsy to tamponade the lobe, assist clot formation and, more importantly, prevent overspill of blood into the adjacent lobes and the contra-lateral lung. This is particularly important as impaired ventilation and gas exchange from clots formed in other areas of the lung in a patient with pre-existing lung disease is the main cause of cardio-respiratory arrest rather than the blood loss itself. In our opinion, the use of balloon blockers is a much better option than using a second bronchoscope. However, the placement of balloon blockers may be difficult in some cases, and the technique also requires training and standardisation of the clinical practice $[3,4,7]$.

The feasibility study published in this edition of Respiration by Franke et al. [13] describes the use of a new 1.1$\mathrm{mm}$ thin cryoprobe (ERBE Elektromedizin $\mathrm{GmbH}$ ) to perform TBLC with a $2.6-\mathrm{mm}$ guide sheath to allow removal of the cryobiopsies directly through the bronchoscope working channel. The authors show in an ex-vivo animal lung that the new mini-cryoprobe obtains alveolar parenchyma specimens 2-3 times bigger than with a 1.8$\mathrm{mm}$ alligator forceps. The new miniprobe might help overcome some major disadvantages of the present cryobiopsy technique, especially the need of intubation, and may facilitate bleeding control. However, in vivo and human studies are needed to demonstrate the efficacy and safety of the new probe. Our personal view is that patient safety should be the primary concern, and hence, even with this adapted technique, it may be better to have airway control with an endotracheal tube in place.

TBLC has become a reality that improves the diagnostic power of the traditional TBLB described in the seventies by Levin et al. [14]. However, large multicentre trials are required to determine the role of TBLC in the management algorithm of interstitial lung diseases. Safety concerns and comparisons with surgical biopsy are crucial aspects to be considered in the near future.

\section{References}

1 Babiak A, Hetzel J, Krishna G, Fritz P, Moeller P, Balli T, Hetzel M: Transbronchial cryobiopsy: a new tool for lung biopsies. Respiration 2009;77:1-6.

-2 Pajares V, Torrego A, Puzo C, Lerma E, Gil de Bernabé MA, Franquet T: Transbronchial lung biopsy using cryoprobes. Arch Bronconeumol 2010;46:111-115.

3 Casoni GL, Tomassetti S, Cavazza A, et al: Transbronchial lung cryobiopsy in the diagnosis of fibrotic interstitial lung diseases. PLoS One 2014;9:e86716.

-4 Pajares V, Puzo C, Castillo D, et al. Diagnostic yield of transbronchial cryobiopsy in interstitial lung disease: a randomized trial. Respirology 2014;19:900-906.

5 Hagmeyer L, Theegarten D, Wohlschläger J, Treml M, Matthes S, Priegnitz C, Randerath WJ: The role of transbronchial cryobiopsy and surgical lung biopsy in the diagnostic algorithm of interstitial lung disease. Clin Respir J 2015. DOI: 10.1111/crj.12261.

\footnotetext{
Fruchter O, Fridel L, Rosengarten D, Raviv Y, Rosanov V, Kramer MR: Transbronchial cryo-biopsy in lung transplantation patients: first report. Respirology 2013;18:669-673.

-7 Hernández-González F, Lucena CM, Ramírez $\mathrm{J}$, et al: Cryobiopsy in the diagnosis of diffuse interstitial lung disease: yield and cost-effectiveness analysis. Arch Bronconeumol 2015; 51:261-267.

$\checkmark 8$ Hetzel J, Eberhardt R, Herth FJ, et al: Cryobiopsy increases the diagnostic yield of endobronchial biopsy: a multicentre trial. Eur Respir J 2012;39:685-690.

-9 Hetzel J, Hetzel M, Hasel C, Moeller P, Babiak A: Old meets modern: the use of traditional cryoprobes in the age of molecular biology. Respiration 2008;76:193-197.

0 Raghu G, Collard HR, Egan JJ, Martinez FJ, et al:. An official ATS/ARS/JRS/ALAT statement: idiopathic pulmonary fibrosis: evidence-based guidelines for diagnosis and management. Am J Respir Crit Care Med 2011;183:788-824.

11 Gershman E, Fruchter O, Benjamin F, et al: Safety of cryo-transbronchial biopsy in diffuse lung diseases: analysis of three hundred cases. Respiration 2015;90:40-46.

12 Poletti V, Hetzel J: Transbronchial cryobiopsy in diffuse parenchymal lung disease: need for procedural standardization. Respiration 2015;90:275-278.

13 Franke K-J, Linzebold W, Nuessle D, Enderle M, Boesmueller H, Nilius G, Hetzel J: A new tool for transbronchial cryobiopsies in the lung: an experimental feasibility ex-vivo study. Respiration 2016;91:228-234.

14 Levin DC, Wicks AB, Ellis JH Jr: Transbronchial lung biopsy via the fiberoptic bronchoscope. Am Rev Respir Dis 1974;110:4-12.
} 